\title{
Cultura ambiental de los habitantes del distrito de Wánchaq de la Región Cusco 2015
}

\author{
Doctora Arminda Margarita Gibaja Oviedo
}

\section{RESUMEN}

El objetivo del estudio fue determinar el nivel de conocimientos sobre cultura ambiental de los habitantes del Distrito de Wánchaq, de la Región Cusco.

Para dicho efecto, se analizaron los indicadores de agua, aire y suelo; considerando que son los componentes básicos y fundamentales para contar con un ambiente relativamente saludable y conocer cómo contribuye con dicho propósito cada una de las personas que habitan en el lugar de estudio.

Se utilizó una encuesta con 60 preguntas — 20 por cada indicador —. La muestra fue de 397 personas de una población de 59134 habitantes (Censo 2007), instrumento que fue sometido al juicio de expertos.

Los datos han sido procesados por el paquete estadístico SPSS, adecuado para las investigaciones en ciencias sociales y otras ramas del conocimiento.

Los resultados obtenidos han permitido conocer el nivel de conocimientos sobre cultura ambiental del distrito en cuestión, el mismo que ha sido de nivel medio. Con relación a los indicadores 'agua' y 'aire', el estudio ha determinado que también alcanzan un nivel medio, sin embargo, en el componente 'suelo' se ha alcanzado el nivel alto.

Considerando la información obtenida a través de fuentes escritas, sabemos que la población de dicho sector de la ciudad de Cusco, tiene estudios de nivel superior y técnico, hecho que también se ha reflejado en la investigación y permite demostrar que la educación juega un rol importante para la formación de una adecuada cultura ambiental.

Palabras clave: Cultura ambiental, agua, aire, suelo 


\section{ABSTRACT}

The study main goal was to establish the knowledge level related to the Wánchaq district inhabitants environmental culture in Cusco region. For such purpose, water, air and soil indicators were proposed to be analyzed, considering that they are the basic and fundamental components to have a relatively healthy environment. It was also proposed the analysis about how every person who lives within the identified area contributes with such purpose.

It was convenient to use a 60 questions survey, 20 per each indicator. The sample was 397 people out of a 59134 inhabitant's population (2007 Census), this instrument has been evaluated by experts. The data has been processed through SPSS statistical software, suitable for research in social sciences and other investigation fields.

The obtained results have allowed to obtain the knowledge level related to the environmental culture within the mentioned district limits which were at a Medium level.

In relation to water and air indicator, the study has determined that they also rose to a medium level; however, the soil component reached a high knowledge level.

Based on information obtained through several written sources, we know that the population in such city sector has superior and technical studies. This fact also demonstrates that education plays an important role for the adequate environmental culture construction.

Key words: Environmental Culture, water, air, soil. 


\section{INTRODUCCIÓN}

El presente trabajo de investigación estuvo orientado a conocer el nivel de cultura ambiental de los habitantes del Distrito de Wánchaq de la Región Cusco.

El espacio geográfico objeto de estudio presenta problemas relacionados al medio ambiente producto, en especial, de la actividad antrópica que representa un peligro para la vida, el equilibrio de la naturaleza y la convivencia social. Todo esto se debe a la ausencia de una adecuada cultura ambiental que ayude a conservar y proteger nuestro entorno.

El estudio pretendió aportar información respecto a si nosotros — seres humanos, desde el lugar que ocupamos en nuestra sociedad-, somos capaces de promover valores comunitarios con el fin de mejorar nuestra calidad de vida considerando que estamos frente al cambio climático que está afectando seriamente el medio ambiente.

La realidad actual nos muestra la crisis que sufre el mundo y, dentro de él, podemos mencionar la escasez del líquido elemento dulce, deterioro de la capa de ozono, cambio climático, deforestación, pérdida de la biodiversidad y suelo fértil; entre otros.

La gran mayoría de los ciudadanos no están muy enterados de los problemas que venimos atravesando, relacionados especialmente con el agua, aire y suelo; elementos importantes para la vida y no tienen clara la idea de cómo participar o contribuir desde el espacio doméstico o público con la protección de los mismos.

Una adecuada cultura ambiental formada - a través de la educación no formal o informal, así como de la educación formal-, contribuirá al mantenimiento de un ambiente sano procurando reducir las fuentes de contaminación.

Es conveniente dar a conocer a las instancias competentes: municipalidades, las ONG, sociedad civil organizada, clubes vecinales, etc., los resultados de esta clase de trabajos, con el objetivo primordial de construir un nuevo tipo de relación entre la sociedad y la naturaleza, además, conseguir el mejoramiento en la calidad de vida. De esta manera, el desarrollo permanente de una cultura ambiental contribuirá en beneficio de la sociedad y de cada uno de nosotros. 
Para el marco teórico se tomó como puntos de referencia diversos trabajos sobre cultura ambiental desarrollados en universidades de diferentes países como Cuba, Colombia, México. Temas relacionados con la comunicación ambiental, problemática ambiental. Se cuenta como herramienta para el fortalecimiento, diagnóstico comunitario y otros, que enfocan la importancia de la cultura ambiental, a la participación ciudadana en favor del ambiente, campañas en contra de productos que perjudiquen el ambiente, la educación como instrumento para lograr el cambio y un comportamiento ecológico adecuado; involucrar a la familia y la comunidad en el cuidado de los recursos como el agua, aire, entre otros; sensibilización para la formación de una cultura ambiental y minimizar los impactos; construcción de una cultura en pro del ambiente.

El desarrollo de investigaciones como la presente, tiene el propósito de hacer conocer sobre el nivel de conocimientos de cultura ambiental de los habitantes del distrito de Wánchaq, a las autoridades de diferentes sectores y especialmente a los municipios distrital y provincial a la población en general, a través de su divulgación por medio de la distribución de folletos, conversatorios, talleres, para lograr acciones específicas encaminadas a proteger el ambiente.

\section{CARACTERÍSTICAS AMBIENTALES DEL DISTRITO DE WÁNCHAQ REFERIDAS AL AGUA, AIRE Y SUELO}

\section{La contaminación del agua en el distrito de Wánchaq}

El agua en su ciclo natural tiene gran capacidad de purificación pero, debido a su abundancia

- y que de manera natural se regenera-, se convierte en el lugar donde se puede arrojar todos los desechos que son el resultado de las actividades humanas. Estos residuos están constituidos por aguas servidas, desechos orgánicos y químicos, detergentes que podemos encontrarlos en cantidades mayores o menores y que son dañinos para la salud y la vida de las personas.

Según el Informe Regional (Cusco, 2012), la provincia de Cusco junto con las provincias Canchis, Paucartambo y Quispicanchis, son las que presentan mayor porcentaje de contaminación hídrica debido a los residuos sólidos. La fuente mayor de captación para el abastecimiento de agua en la provincia de Cusco es de manantiales, siendo el 60,7\% de la población que consume el líquido elemento de sistemas de gravedad con tratamiento, así se indica en el Informe Regional. 
La contaminación hídrica, de acuerdo al Plan de Desarrollo de la Municipalidad de Wánchaq (2013), se focaliza en las cuencas del río Huatanay y del riachuelo Cachimayo, constituyéndose - el primero - en el mayor foco contaminante, no solo del Distrito de Wánchaq, sino de la Provincia de Cusco, al haberse convertido en el mayor vertedero de las aguas servidas $(70 \%)$ de la ciudad de Cusco, habida cuenta la insuficiente capacidad del sistema colector de la planta de tratamiento de San Jerónimo, además de ser y servir como botadero abierto de basura y residuos sólidos. El Instituto de Manejo de Agua y Medio Ambiente del Cusco, estima que el $12 \%$ de la basura generada en la ciudad del Cusco se vierte al río Huatanay, calificando el nivel de su contaminación como 'severa.'

El año de 2014, la Autoridad Nacional del Agua (ANA) Cusco, realizó el registro e identificación de las fuentes contaminantes de la cuenca del río Huatanay, identificando los puntos de vertimiento de efluentes líquidos y residuos sólidos. Son varios los ríos ubicados en la cuenca del río Huatanay donde se encuentra la ciudad del Cusco, y varios de sus distritos. Este río cruza la cuenca de noroeste a este y tiene varios efluentes, entre ellos los ríos Saphy, el Choquechaca, Huancaro; entre otros. El vertimiento de aguas residuales al río Watanay se realiza en 29 lugares o sectores. Es de lamentar que muchos de ellos no han recibido el tratamiento de las aguas residuales (PTAR) antes de la descarga, lo que contamina el aire, el agua y el suelo. En la actualidad, se cuenta con una planta de tratamiento de aguas residuales ubicada en el distrito de San Jerónimo.

El año 2015, la Oficina de Gestión Ambiental de la Municipalidad Distrital de Wánchaq, realizó un estudio sobre el vertimiento de efluentes líquidos y residuos sólidos domésticos en la margen izquierda de los ríos Watanay y Cachimayo, por parte de los pobladores de las diferentes urbanizaciones que se encuentran a lo largo de los ríos indicados, estos residuos caen en su mayor parte al agua, contaminándola.

\section{La contaminación del aire en el distrito de Wánchaq}

Se ha determinado que la contaminación atmosférica es producida, principalmente, por la creciente proliferación del parque automotor de vehículos antiguos superior a los 10 años y que son los que generan gases tóxicos. Esta situación es más crítica en los principales corredores vehiculares como las avenidas: De la Cultura, Huayruropata y La Infancia; que 
registran índices superiores a 900 veh/hora, así se indica en el Plan de Desarrollo Concertado de la Municipalidad Distrital de Wánchaq, (Wánchaq, 2013).

Es necesario indicar también, que está presente de forma permanente la contaminación acústica en el distrito, la que está referida principalmente al ruido ocasionado por el aterrizaje y despegue de los aviones en el Aeropuerto Velazco Astete del Cusco, cuya infraestructura se encuentra localizada dentro de la demarcación territorial de la Municipalidad de Wánchaq. La Municipalidad Provincial del Cusco en el año 2013, a través de la Gerencia de Desarrollo Urbano y Rural y; la Subdirección de Ordenamiento Territorial, ejecutaron el proyecto de mejoramiento del servicio de ordenamiento territorial sobre la calidad del aire en la cuenca atmosférica del valle del Cusco. El trabajo levantó la línea de base de la calidad del aire: material particulado menor a $10 \mu$ (micras) y metales pesados; desde junio de 2012 a junio de 2013, habiendo alcanzado los siguientes resultados:

Sobre la calidad del aire, se toma importancia desde el año 2001 fecha en la que se emite el D.S. 074.2001-PCM, dando a conocer los estándares nacionales de calidad de aire (ECA) y el D.S. 047-2001-MTC, que indica los límites máximos permisibles de emisiones de contaminantes para vehículos automotores que circulan en la red vial. El monitoreo de la calidad de aire la realiza la Dirección Regional de Salud Ambiental, Cusco-DIRESA. El estudio contó con la participación de las municipalidades distritales de Lucre, Oropeza, Andahuaylillas, Saylla, San Jerónimo, San Sebastián, Wánchaq, Cusco, Santiago, Qorqa, Poroy y Anta.

De las muestras tomadas sobre material particulado, se determinaron siete (7) puntos críticos dentro de la cuenca atmosférica de Cusco y zonas contiguas. Entre estos 7, tenemos a dos (2) que se encuentran en el distrito de Wánchaq, cuyos niveles de PM10 superan el estándar de calidad ambiental del aire respecto al PM10 de $150(\mu \mathrm{g} / \mathrm{m} 3) / 24$ horas, estos son:

- CC HH Hilario Mendívil, 219

- Parque Pukllaycancha 201

Asimismo, el estudio determinó nueve (9) puntos críticos que están próximos a los estándares de calidad de aire, ECA $150(\mu \mathrm{g} / \mathrm{m} 3) / 24$ horas, por encima del $50 \%$ del ECA, es decir en el umbral, lo que indicaba tomar medidas de prevención y regulación para evitar el incremento 
de la concentración del material particulado en la cuenca atmosférica en estudio. En este caso, para el distrito de Wánchaq tres (3) puntos críticos:

$\begin{array}{lll}- & \text { Frontis de la Municipalidad Distrital de Wánchaq } & 147 \\ \text { - } & \text { Centro de Salud Ttío-Wánchaq } & 124 \\ \text { - } & \text { Avenida Huayruropata, Iglesia Adventista } & 102\end{array}$

El estudio, si bien es cierto incluyó los puntos de concentración máxima de metales pesados, no lo hizo con el Distrito de Wánchaq, y se determinó que el material particulado menor de $10 \mu$ PM10 como el que genera mayor impacto en la salud pública, ocasionado por la combustión de gas, material particulado y polvo fugitivo del parque automotor, de chimeneas, pollerías y pizzerías; así como producto de las construcciones, fábricas de tejas y ladrillos, quema de residuos sólidos, rastrojo, pastos, tráfico aéreo y ferroviario.

Los contaminantes del aire producen efectos negativos en la salud de las personas, indicaremos algunos de ellos:

- $\quad$ Dióxido de azufre ( $\left.\mathrm{SO}_{2}\right)$, ocasiona traqueítis, bronquitis.

- $\quad$ Dióxido de nitrógeno $\left(\mathrm{NO}_{2}\right)$, bronquitis, pulmonía.

- $\quad$ Monóxido de carbono (CO), inhabilita el transporte de oxígeno a las células.

- $\quad$ Ozono $\left(\mathrm{O}_{3}\right)$, irrita el sistema respiratorio, asma.

- $\quad$ Plomo $(\mathrm{Pb})$, material particulado con diámetro menor de $10 \mu$, o menor a $2,5 \mu$.

Igualmente, se ha determinado que la inversión térmica de subsidencia producida entre los meses de mayo a setiembre aumenta la concentración de los contaminantes.

El material particulado, además de afectar a la salud, va deteriorando el patrimonio cultural como son: los edificios, esculturas, pinturas, obras en metal, madera, etc. La presencia de dióxido de azufre $\left(\mathrm{SO}_{2}\right)$ y dióxido de nitrógeno $\left(\mathrm{NO}_{2}\right)$ en la atmósfera ocasionan la lluvia ácida que deteriora el patrimonio cultural.

El mismo documento trae una síntesis del diagnóstico social de la ciudad del Cusco, y en sus cuadros muestra el índice de desarrollo humano de la Provincia del Cusco y el Distrito de Wánchaq, que alcanzan los niveles más altos con relación a los demás de la provincia, como 
se puede apreciar en el cuadro comparativo que presentamos de los años 2005 y 2012, en el cual se aprecia, cómo se aumentaron los niveles de desarrollo humano en el distrito que nos ocupa.

CUADRO COMPARATIVO DEL ÍNDICE DE DESARROLLO HUMANO DEL DISTRITO DE WÁNCHAQ

Cuadro N. ${ }^{\circ} 1$

\begin{tabular}{|l|l|l|}
\hline DISTRITO DE WANCHAQ & $\begin{array}{l}\text { Año } \\
2005\end{array}$ & $\begin{array}{l}\text { Año } \\
2012\end{array}$ \\
\hline IDH & 0,5555 & 0,6769 \\
\hline Esperanza de vida al nacer & 63,80 & 74,48 \\
\hline Analfabetismo \% & 91,30 & 98,88 \\
\hline Escolaridad \% & 92,1 & 92,66 \\
\hline Logro educativo \% & 91,6 & 96,81 \\
\hline Ingreso familiar per cápita & 250,9 & 526,2 \\
\hline
\end{tabular}

Fuente: Elaboración propia

ÍNDICE DE DESARROLLO HUMANO EN LA PROVINCIA DELCUSCO

CUADRO N. ${ }^{\circ} 2$

CONSOLIDADO SOBRE ÍNDICE DE DESARROLLO HUMANO EN LA PROVINCIA DEL CUSCO

\begin{tabular}{|l|r|r|r|r|r|r|}
\hline Distrito & \multicolumn{1}{|c|}{ IDH } & $\begin{array}{c}\text { Esperanza } \\
\text { de vida al } \\
\text { nacer, } \\
\text { años }\end{array}$ & $\begin{array}{r}\text { Alfabetismo } \\
\%\end{array}$ & Escolaridad & $\begin{array}{c}\text { Logro } \\
\text { Educativo }\end{array}$ & $\begin{array}{r}\text { Ingr.fam. } \\
\text { Per cápita }\end{array}$ \\
\hline Cusco & 0,6535 & 74,15 & 96,57 & 91,23 & 94,79 & 434,2 \\
\hline Ccorca & 0,5150 & 72,68 & 62,24 & 86,65 & 70,37 & 131,4 \\
\hline Poroy & 0,5968 & 73,25 & 88,82 & 89,24 & 88,96 & 234,5 \\
\hline $\begin{array}{l}\text { San } \\
\text { Jerónimo }\end{array}$ & 0,6481 & 74,01 & 95,26 & 91,06 & 93,87 & 425,1 \\
\hline $\begin{array}{l}\text { San } \\
\text { Sebastián }\end{array}$ & 0,6505 & 74,08 & 96,12 & 90,46 & 94,23 & 429,7 \\
\hline Santiago & 0,6382 & 74,01 & 95,04 & 89,92 & 93,33 & 374,6 \\
\hline Saylla & 0,6156 & 73,51 & 93,12 & 91,78 & 92,67 & 265,3 \\
\hline Wánchaq & 0,6769 & 74,48 & 98,88 & 92,66 & 96,81 & 526,2 \\
\hline
\end{tabular}

Fuente: Elaboración propia 
El año 2012, la Dirección de Evaluación (OEFA), realizó una evaluación rápida del ruido ambiental en la ciudad de Cusco, para dicho efecto, determinaron las siguientes zonas: de protección especial, zona residencial, zona comercial, zonas mixtas, zonas críticas de contaminación sonora; los horarios considerados fueron el diurno y nocturno. Se determinaron 32 lugares para monitorear con sonómetros: avenidas, cruce de calles, inmediaciones de fábricas de ladrillos. Los ruidos producidos por el transporte automotor se constituyen en una de las mayores fuentes de contaminación acústica en la ciudad. Este ruido fue clasificado en tres tipos: el de propulsión (el motor y el sistema de escape asociado), el de rodadura entre la pista y las llantas y, el aerodinámico. Las conclusiones a las que llegaron fueron las siguientes:

Los niveles de ruido obtenidos en los 32 sitios monitoreados estaban entre un mínimo de 66,6 $\mathrm{dB}$ (decibeles) y un máximo de 72,2 dB; la zona de ruido ambiental más alto estaba ubicada en el cruce de la Avenida de la Cultura y Calle Retiro (distrito de Wánchaq), con una medición de 72,2 dB, valor aproximado a $80 \mathrm{~dB}$, siendo considerada como 'zona crítica', los emisores son: transporte público, particular, interprovincial, la congestión de las vías públicas y el uso de las bocinas. (Informe N. ${ }^{\circ}$ 568-2012-OEFA/DE. (Evaluación rápida de ruido ambiental en la ciudad de Cusco).

En el año 2014 (Medina, 2015), realizó un estudio sobre los factores determinantes de contaminación del aire con monóxido de carbono (CO) en la localidad del Cusco, con 40 mediciones en diferentes calles y avenidas de la ciudad con sus variables, altura de edificación, ancho de calle, número de vehículos que circulan en un lapso de 30 minutos; de la misma manera, se consideraron dos sectores: calles del centro histórico y calles de la zona periférica al centro histórico, que consideraba al distrito de Wánchaq. Los resultados obtenidos para el centro histórico indican que la contaminación ambiental del aire con monóxido de carbono (CO) supera los estándares mínimos permitidos, mientras que para las zonas periféricas - donde se encuentra el distrito de Wánchaq, que es el sector de nuestro estudio-, la contaminación del aire con monóxido de carbono está por debajo de los estándares mínimos. 


\section{La contaminación del suelo en el distrito de Wánchaq}

Silva y Correa F. (2009), mencionan que un suelo puede degradarse cuando se acumulan sustancias tóxicas a niveles que repercuten negativamente en su comportamiento, provocando la pérdida parcial o total de la productividad.

Asimismo, el suelo es un componente muy específico de la biosfera porque actúa como amortiguador natural controlando el transporte de elementos y sustancias químicas a la atmósfera, la hidrosfera y la biota. Por tanto, el mantenimiento de las funciones ecológicas del suelo es responsabilidad de la humanidad, así lo manifiesta (Seoanez Calvo, 1999, pág. 64).

En el Plan de desarrollo concertado de la Municipalidad Distrital de Wánchaq al 2013, el manejo de los residuos sólidos que produce la actividad económica llega a las 43257 TM/día, donde el 20,20 \% es de origen orgánico producido por residuos sólidos, tierra colmatada, vegetación existente en las calles, avenidas y parques, además, que el distrito no cuenta con un relleno sanitario propio utilizando el de la ciudad de Cusco, ubicado en el sector conocido como Haquira del Distrito de Santiago. Los residuos sólidos del Distrito de Wánchaq, en el año 2010 alcanzó las 16 959.00 TM anuales (propuesta: «Plan de manejo de residuos sólidos de la Municipalidad Provincial del Cusco 2011»). Asimismo, la Municipalidad de Cusco cuenta con una planta de segregación de residuos sólidos urbanos y otra planta de tratamiento de residuos orgánicos, ubicados en la zona de Rayallaqta-Andahuaylillas. En la primera, se realiza la separación y clasificación de los residuos en 'orgánicos', 'inorgánicos' y los que ‘ya no sirven'. Los primeros se los coloca en depósitos de plástico con tapa y luego son trasladados a la planta de tratamiento de Rayallaqta. Los inorgánicos que pueden ser reciclados son: el papel, plásticos duros, botellas de plásticos, latas; una vez clasificados son envasados para su venta y serán utilizados como materia prima para producir nuevas latas de metal, papel reciclado, nuevos plásticos y telas polar, entre otros.

Los residuos orgánicos son tratados en la planta de tratamiento de Rayallaqta, cuyo propósito es la preparación de humus a través de dos procedimientos: el compostaje y la lombricultura, luego, todos los residuos son llevados al relleno sanitario de Haquira. 


\section{La conservación del ambiente y su importancia}

Para desarrollar el tema es necesario hablar de conservación, es decir, nos estamos refiriendo al 'gran sistema ambiental integrado por la atmósfera, hidrósfera, litósfera, biósfera y la tecnosfera en todas las zonas'; hasta las más prístinas han sido intervenidas por el hombre y muestran evidencias de acciones de preservación o conservación como lo menciona (Giannuzzo, 2010). Igualmente, indica que el cambio climático es otro ejemplo de cómo la actividad humana daña la naturaleza y la interdependencia de los subsistemas: atmósfera, litosfera y los demás.

La conservación es el conjunto de políticas y medidas para mantener las condiciones que propicien la evolución y continuidad de los ecosistemas y hábitat naturales, así como conservar las poblaciones viables de especies en sus entornos naturales y los componentes de la biodiversidad fuera de su entorno natural. La acción del hombre sobre la naturaleza a través del tiempo, ha logrado que desaparezcan diferentes especies de animales y plantas, otros están camino a su extinción; se ha contaminado el recurso hídrico de mares y ríos con deshechos cloacales, plástico y petróleo, así como, el suelo y las aguas con pesticidas y el aire con la presencia de gases tóxicos, el smog.

Proteger el medio ambiente significa también, cuidar algunos lugares geográficos, culturas originarias que mantienen sus costumbres, tradiciones, música, danza, idioma, artesanía y que son un emporio de riqueza de cultura inmaterial y material, tangible como la arquitectura, pintura, escultura; que son parte de la creatividad del hombre, de su cultura. Para el caso de la arquitectura histórica, la preservación consiste en resguardar su integridad prohibiendo su demolición y garantizando que el edificio no tenga cambios estructurales. Por ello, la preservación del patrimonio cultural de un pueblo es responsabilidad de toda persona como miembro integrante de una sociedad.

La preservación está orientada a evitar las actividades contaminantes, utilizar energías limpias y renovables — solar, eólica, entre otras-, efectuar campañas de reciclaje, disminución de la emisión de dióxido de carbono a la atmósfera.

Para evitar la destrucción del medio ambiente, así como la supervivencia de los seres vivos

y un adecuado desarrollo humano (Cuervo Álvarez, 2012), manifiesta que es conveniente 
tomar medidas necesarias y urgentes, entre ellas menciona: hacer uso adecuado de los recursos naturales, no utilizarlos de manera indiscriminada y hacerlos sostenibles $\mathrm{y}$ sustentables.

En la tarea de protección medioambiental, es conveniente, que todos los actores de la sociedad tomen conciencia y se involucren antes de que sea demasiado tarde. Si queremos tener un ambiente todavía habitable, es conveniente, que desde el hogar y en nuestro actuar cotidiano realicemos acciones de cuidado del medio ambiente $\mathrm{y}$, desde las organizaciones comunales, clubes, sociedad civil organizada, etc., exigir a los estados y a sus gobernantes que promuevan, a través de diferentes mecanismos —especialmente la educación-, el cuidado de nuestra casa y que se tomen medidas para controlar el accionar de las industrias, empresas y de la comunidad científica cuidando el bienestar humano y no solo el lucro de los grupos económicos de poder.

El ser humano no es el único que ocupa la tierra, junto con él están otros seres vivos con los cuales convive e interactúa de modo permanente, puesto que todos tenemos que subsistir y los elementos básicos como el agua, aire, suelo, flora y fauna, son sumamente importantes, porque permiten mantener el equilibrio adecuado entre todos los seres vivos y los agentes fisicoquímicos.

Muñoz, M. (2010) tomando a lo indicado en el «Informe Brundtland» de la Comisión Mundial de Medio Ambiente y Desarrollo de las Naciones Unidas, aduciendo que «El futuro está amenazado», indica lo siguiente:

La tierra es una, pero el mundo no lo es. Todos dependemos de una biosfera para mantenernos con vida. Sin embargo, cada comunidad, cada país lucha por sobrevivir y prosperar sin preocuparse de los efectos que causa en los demás. Algunos consumen los recursos de la tierra a un ritmo que poco dejará para las generaciones futuras. Otros, muchos más numerosos, consumen muy poco y arrastran una vida de hambre y miseria, enfermedad y muerte prematura. Los pueblos pobres se ven obligados a utilizar en exceso los recursos del medio ambiente para sobrevivir al día, y el empobrecimiento de su medio ambiente contribuye a acentuar su indigencia y a hacer aún más difícil e incierta su supervivencia. (Brundtland, 1987).

La sociedad humana tiene el derecho de contar con un ambiente saludable y llevar una vida digna. Para ello debe vivir en armonía con su naturaleza, su ambiente, satisfaciendo sus 
necesidades, pero igualmente, tiene la obligación ineludible de protegerlo, conservarlo y hacerlo sostenible para el uso y disfrute de las generaciones presentes y de las venideras.

\section{Cultura y cultura ambiental}

Cultura, para la Organización de las Naciones Unidas para la Educación la Ciencia y la Cultura (UNESCO, 2012), es el conjunto de rasgos distintivos espirituales y humanos que caracterizan a una sociedad o grupo social, engloba no solo las artes y las letras, sino también los modos de vida, los derechos fundamentales del ser humano, los sistemas de valores, las creencias.

Tomando a Bonfil B.:

[...] la cultura es el conjunto de símbolos, valores, actitudes, habilidades, conocimientos, significados, formas de comunicación y organización sociales y bienes materiales que hacen posible la vida de una sociedad determinada y le permiten transformarse y reproducirse como tal, de una generación a las siguientes. (Bonfil Batalla, 2004, pág. 37)

La cultura también es la forma cómo se da respuesta a los diferentes problemas que la humanidad tiene, en síntesis: son todas y cada una de las actividades que realizamos y que están plasmados en elementos materiales e inmateriales, costumbres, tradiciones, mitos, leyendas, conocimientos ancestrales creencias, arte, moral, derecho, hábitos, actitudes; todos adquiridos por el hombre como miembro de una sociedad.

Otras definiciones consideran a la cultura todo lo que es no biológico y transmitido socialmente en una sociedad, incluyendo los esquemas de conducta artística, social, ideológica y religiosa y las técnicas para dominar el mundo circundante, (Winick, 1989).

El antropólogo (Herskovits, 2000, pág. 22), informa que la cultura es algo que puede ser aprendida, estructurada, que es divisible en aspectos dinámicos y variables, que emerge de todos los componentes de la existencia humana. Estos profesionales entienden que la cultura es el conjunto total de los actos humanos en una comunidad dada, ya sean prácticas económicas, artísticas, científicas u otras, considerando además, que toda práctica humana que supere la naturaleza biológica es una práctica social.

(Mac Gregor, 2005), manifiesta que la cultura también tiene que ver con la identidad, con todo aquello que se tiene que defender como propio; la identidad de un grupo o de un pueblo se enriquece permanentemente y se renueva con aportaciones de los individuos que la 
confirman. La identidad de un pueblo es pasado, historia y memoria colectiva. Es necesario defender lo propio, pero también apropiarse de lo ajeno que sirva, agregue y enriquezca la identidad.

En cuanto se refiere a la cultura ambiental, se entiende como aquella postura ante la vida que nos permite cuidar y preservar nuestro medio ambiente, es un asunto de interés para todo el mundo.

(Guadarrama, P y N. Pelegrín, 1990) manifiestan que la cultura ambiental se expresa en esa segunda naturaleza construida por el hombre, las relaciones no solo materiales sino también espirituales que se establecen, formando parte de ese concepto grande que es Medio Ambiente.

(Mata, 2004), nos dice que:

La definición y análisis de la cultura ambiental que adopta una sociedad —o grupo en particulardebe partir de la observación de las conductas o el comportamiento ambiental que permite la comprensión de los diferentes preceptos culturales a lo largo del tiempo, con relación al ambiente, por ejemplo las prácticas agrícolas, los estilos de consumo, la aplicación de políticas ambientales, la conducta ciudadana, el manejo de desechos, la conducta individual. (Mata, 2004).

Quienes conocen y practican la cultura ambiental, son personas que conocen las bondades y problemática de su comunidad y su medio ambiente, que participan de la cultura y tradición de su pueblo, les gusta explorar la naturaleza, dinamizar y estimular los espacios comunitarios para la recuperación, conservación y gozo del medio ambiente y del conocimiento local, tienen capacidad de gestión y convocatoria, principalmente tienen humildad y sensibilidad para percibir las necesidades de los integrantes de su comunidad, así lo sostienen (Amezcua, Cardelia y Prodanza, C., 2006).

Para Bayón en (Miranda, 2011, pág. 41), la cultura ambiental está sustentada en la relación del hombre con su medio ambiente, y en dicha relación está implícito el conjunto de estilos, costumbres y condiciones de vida de una sociedad con una identidad propia basada en tradiciones, valores y conocimientos.

De la misma manera, la cultura ambiental también es la manera de pensar, sentir y actuar en nuestro ambiente que está en constante cambio, es el uso de los recursos naturales con responsabilidad, haciéndolos sostenibles para el disfrute de las generaciones futuras. 


\section{Nivel de cultura ambiental}

El vocablo conocido como 'nivel', se utiliza para describir la horizontalidad de una determinada cosa. Por ejemplo, puede reconocerse un nivel como la altura a la que llega algo o a la que ha sido ubicado. También se acepta como la altura máxima que alcanza la superficie de una sustancia líquida (de allí, por ejemplo, que sea habitual decir frases como «el nivel del río ha crecido»).

El Diccionario de la Lengua Española (Asociación de Academias de la Lengua Española, 2014), define a esta palabra como la medida de una cantidad en relación a una escala específica ("nivel de azúcar en sangre”); la que la presenta como sinónimo de categoría, escalafón o rango ("es un jugador de excelente nivel”); aquella que lo admite como el nombre que se le otorga al instrumento que permite descubrir la diferencia o igualdad de altura que puede existir entre dos puntos; aquella que habla de nivel como la semejanza o similitud en cualquier línea o especie.

En el caso de nivel cultural, presenta como el grado o altura que se logra en determinadas cuestiones del ámbito social ("se trata de una persona de buen nivel cultural”).

El concepto que nos ocupa, también se emplea con mucha frecuencia en el ámbito de la educación. Así, se habla de niveles educativos cuando nos referimos a las diversas especialidades: bachillerato, licenciatura, maestría, doctorado; ellos nos indican la posición formativa en la que se encuentra una persona con respecto a otra u otras.

La cultura es muy variada, dinámica y, por consiguiente, muy cambiante por la variedad de los agentes implicados, lo heterogéneo de los territorios y la diversidad de las manifestaciones culturales, por lo cual su análisis es complejo y es muy dificultoso contar con un sistema de información único con indicadores globales y consensuados, más allá de los descriptivos, así lo afirma (Carrasco Arroyo, 2006).

Las características observadas de un determinado comportamiento de una población se expresa de dos maneras distintas que corresponden a dos tipos de variables, y, que a su vez, generan dos clases de datos: categóricas o cualitativas y numéricas o cuantitativas. La 'categórica' se refiere a cualidades pueden ser: opiniones, percepciones de la población sobre algo y que se pueden medir en niveles nominales $u$ ordinales. 
Para el planteamiento del estudio se trabajó con una variable cualitativa que es 'cultura ambiental', a la que se ha asignado indicadores que nos permitirá conocer a la población en estudio considerando la edad, el nivel educativo, el sexo, el lugar de trabajo; los cuales tienen relación directa con la preservación del ambiente en sus elementos más importantes como el agua, el aire y el suelo. La medición fue de nivel nominal, proporcionando información relevante para que se puedan tomar decisiones en el ámbito del comportamiento de las personas respecto a su ambiente.

El indicador va a constatar hechos, comportamientos y formas de vida (pasadas, presentes) y también, da cuenta de los patrones culturales de los ciudadanos. Para medir se puede explicitar las preferencias frente a los valores, sin necesidad de expertos o intérpretes. En la investigación realizada por (Isaac-Márquez, Ricardo; Salavarría García, Oswaldo Orlando; Eastmond Spencer, Amarella; Ayala Arcipreste, María Esther; Arteaga Aguilar, Marco Antonio; Et. Al., 2011), sobre cultura ambiental en estudiantes de bachillerato en la Universidad Autónoma de Campeche, México, describe que la cultura ambiental es el conjunto de actitudes, intenciones de comportamiento y conocimientos ambientales que posee una persona. En la discusión señala que el estudio ha demostrado que los estudiantes de la Universidad Autónoma de Campeche, ha dado como resultado que tienen un nivel de cultura ambiental relativamente exigua, haciendo que la investigación haya planteado las categorías de alto, medio y bajo. Para la investigación presente se ha considerado los mismos niveles, tomando como referencia dicha investigación, la que se asume como válida.

\title{
RESULTADOS:
}

\author{
Tabla N. ${ }^{\circ} 1$ \\ COMPOSICIÓN DE LA MUESTRA SEGÚN DATOS DE INFORMACIÓN
}




\begin{tabular}{lcc}
\hline Datos generales & $\mathbf{N .}^{\circ}$ & $\mathbf{\%}$ \\
\hline \hline 1. Edad & & \\
15 a 32 años & 172 & 43,3 \\
33 a 50 años & 149 & 37,5 \\
51 a 68 años & 67 & 19,9 \\
69 a 85 años & 9 & 2,3 \\
\hline 2. Género & 192 & \\
Femenino & 205 & 48,4 \\
Masculino & & 51,6 \\
\hline 3. Nivel educativo & 1 & \\
Primaria & 64 & 0,3 \\
Secundaria & 324 & 16,1 \\
Superior & 8 & 81,6 \\
Técnico & & 2.0 \\
\hline 4. Lugar de trabajo & 130 & 32,7 \\
Sector público & 90 & 22,7 \\
Sector privado & 104 & 26,2 \\
Independiente & 24 & 6.0 \\
En casa & 49 & 12,3 \\
Otro & $\mathbf{3 9 7}$ & $\mathbf{1 0 0 . 0}$ \\
\hline Total & & \\
\hline
\end{tabular}

Fuente: Encuesta sobre conocimiento de cultura ambiental

En la tabla se observa que la mayor proporción de la muestra de los pobladores del Distrito de Wánchaq fluctúa entre las edades de 15 a 32 años y entre 33 a 50 años en una proporción del 43,3\%; el 37,5 \% son varones, el 81,6\% presentan un nivel educativo superior, y el 32,7 $\%$ trabajan en el sector público. 
En primer término, se realizó un análisis descriptivo utilizando tablas de contingencia entre los niveles de conocimiento sobre cultura ambiental en sus tres componentes agua, aire y suelo, para identificar proporciones altas.

\section{ANÁLISIS SOBRE CULTURA AMBIENTAL SEGÚN EDAD}

Tabla N. ${ }^{\circ} 2$ NIVEL DE CONOCIMIENTO SOBRE CULTURA AMBIENTAL SEGÚN EDAD

\begin{tabular}{lcccccccccc}
\hline & \multicolumn{2}{c}{ Bajo } & \multicolumn{2}{c}{ Medio } & \multicolumn{2}{c}{ Alto } & \multicolumn{2}{c}{ Muy alto } & \multicolumn{2}{c}{ Total } \\
\cline { 2 - 10 } & $\mathrm{N}^{\circ}$ & $\%$ & $\mathrm{~N}^{\circ}$ & $\%$ & $\mathrm{~N}^{\circ}$ & $\%$ & $\mathrm{~N}^{\circ}$ & $\%$ & $\mathrm{~N}^{\circ}$ & $\%$ \\
\hline \hline $\begin{array}{l}15-32 \\
\text { años }\end{array}$ & 26 & $15,1 \%$ & 92 & $53,5 \%$ & 54 & $31,4 \%$ & 0 & $0,0 \%$ & 172 & $100 \%$ \\
$\begin{array}{l}33-50 \\
\text { años }\end{array}$ & 12 & $8,1 \%$ & 77.0 & $51,7 \%$ & 59 & $39,6 \%$ & 1 & $0,7 \%$ & 149 & $100 \%$ \\
$\begin{array}{l}51 \text { a } 68 \\
\text { años } \\
69 \text { a } 85\end{array}$ & 7 & $10,4 \%$ & 31 & $46,3 \%$ & 29 & $43,3 \%$ & 0 & $0,0 \%$ & 67 & $100 \%$ \\
años & 3 & $33,3 \%$ & 2 & $22,2 \%$ & 4 & $44,4 \%$ & 0 & $0,0 \%$ & 9 & $100 \%$ \\
\hline Total & 48 & $12,1 \%$ & 202 & $50,9 \%$ & 146 & $36,8 \%$ & 1 & $0,3 \%$ & 397 & $100.0 \%$ \\
\hline
\end{tabular}

Fuente: Encuesta sobre conocimiento de cultura ambiental

La tabla $\mathrm{N} .^{\circ}$ 2, refleja que los habitantes del distrito de Wánchaq según "grupo etario" presentan en general un nivel de conocimiento 'medio' sobre "cultura medio ambiental", destacando las personas de 15 a 32 años en una proporción del 53,5\%, seguido por los de 33 a 50 años con una proporción del 51,7 \%.

\section{ANÁLISIS SOBRE CULTURA AMBIENTAL SEGÚN GÉNERO}


Tabla N. ${ }^{\circ} 3$ NIVEL DE CONOCIMIENTO SOBRE CULTURA AMBIENTAL, SEGÚN GÉNERO.

\begin{tabular}{ccccccccccc}
\hline & \multicolumn{2}{c}{ Bajo } & \multicolumn{2}{c}{ Medio } & \multicolumn{2}{c}{ Alto } & \multicolumn{2}{c}{ Muy alto } & \multicolumn{2}{c}{ Total } \\
\cline { 2 - 11 } & N. & $\%$ & $\mathrm{~N}^{\circ}$ & $\%$ & $\mathrm{~N}^{\circ}$ & $\%$ & $\mathrm{~N}^{\circ}$ & $\%$ & $\mathrm{~N}^{\circ}$ & $\%$ \\
\hline \hline Femenino & 19 & $10 \%$ & 99 & $52 \%$ & 73 & $38 \%$ & 1 & $1 \%$ & 192 & $100 \%$ \\
Masculino & 29 & $14 \%$ & 103 & $50 \%$ & 73 & $36 \%$ & 0 & $0 \%$ & 205 & $100 \%$ \\
\hline Total & 48 & $12 \%$ & 202 & $51 \%$ & 146 & $37 \%$ & 1 & $0 \%$ & 397 & $100 \%$ \\
\hline
\end{tabular}

Fuente: Encuesta sobre nivel de conocimiento de cultura ambiental

En la tabla N. ${ }^{\circ} 3$, se observa que el nivel de conocimiento sobre 'cultura ambiental' que presentan los pobladores del distrito de Wánchaq es mayormente 'medio', destacando las mujeres cuya proporción es del $52 \%$, en comparación a los varones con un $50 \%$.

\section{ANÁLISIS SOBRE CULTURA AMBIENTAL SEGÚN NIVEL EDUCATIVO}

\section{Tabla N. ${ }^{\circ} 4$ NIVEL DE CONOCIMIENTO SOBRE CULTURA AMBIENTAL SEGÚN NIVEL EDUCATIVO}

\begin{tabular}{lcccccccccc}
\hline \multirow{2}{*}{ Nivel educativo } & \multicolumn{2}{c}{ Bajo } & \multicolumn{2}{c}{ Medio } & \multicolumn{2}{c}{ Alto } & \multicolumn{2}{c}{ Muy alto } & \multicolumn{2}{c}{ Total } \\
\cline { 2 - 11 } & $\mathrm{N}^{\circ}$ & $\%$ & $\mathrm{~N}^{\circ}$ & $\%$ & $\mathrm{~N}^{\circ}$ & $\%$ & $\mathrm{~N}^{\circ}$ & $\%$ & $\mathrm{~N} .{ }^{\circ}$ & $\%$ \\
\hline \hline PRIMARIA & 0 & $0 \%$ & 1 & $100 \%$ & 0 & $0 \%$ & 0 & $0 \%$ & 1 & $100 \%$ \\
SECUNDARIA & 14 & $22 \%$ & 29 & $45 \%$ & 21 & $33 \%$ & 0 & $0 \%$ & 64 & $100 \%$ \\
SUPERIOR & 33 & $10 \%$ & 170 & $52 \%$ & 120 & $37 \%$ & 1 & $0 \%$ & 324 & $100 \%$ \\
TÉCNICO & 1 & $13 \%$ & 2 & $25 \%$ & 5 & $63 \%$ & 0 & $0 \%$ & 8 & $100 \%$ \\
\hline \multicolumn{1}{c}{ Total } & 48 & $12 \%$ & 20 & $51 \%$ & 146 & $37 \%$ & 1 & $0 \%$ & 397 & $100 \%$ \\
\hline
\end{tabular}

Fuente: Encuesta sobre nivel de conocimiento de cultura ambiental

La tabla N..$^{\circ}$, muestra que la mayoría de los habitantes del Distrito de Wánchaq presentan un nivel de conocimiento 'medio' respecto a la 'cultura ambiental', sin embargo, el $63 \%$ de los técnicos presentan conocimientos altos y un habitante que tiene estudios superiores logró un conocimiento muy alto del tema. 
ANÁLISIS SOBRE LA CULTURA AMBIENTAL, SEGÚN LUGAR

DE TRABAJO

Tabla N. ${ }^{\circ} 5$ NIVEL DE CONOCIMIENTO SOBRE CULTURA AMBIENTAL, SEGÚN LUGAR DE TRABAJO

\begin{tabular}{lcccccccccc}
\hline \multirow{2}{*}{$\begin{array}{c}\text { Lugar de } \\
\text { trabajo }\end{array}$} & \multicolumn{2}{c}{ Bajo } & \multicolumn{2}{c}{ Medio } & \multicolumn{2}{c}{ Alto } & \multicolumn{2}{c}{ Muy alto } & \multicolumn{2}{c}{ Total } \\
\cline { 2 - 11 } & $\mathrm{N}^{\circ}$ & $\%$ & $\mathrm{~N}^{\circ}$ & $\%$ & $\mathrm{~N}^{\circ}$ & $\%$ & $\mathrm{~N}^{\circ}$ & $\%$ & $\mathrm{~N}^{\circ}$ & $\%$ \\
\hline \hline Sector publico & 14 & $11 \%$ & 59 & $45 \%$ & 56 & $43 \%$ & 1 & $1 \%$ & 130 & $100 \%$ \\
Sector privado & 8 & $9 \%$ & 50 & $56 \%$ & 32 & $36 \%$ & 0 & $0 \%$ & 90 & $100 \%$ \\
Independiente & 12 & $12 \%$ & 54 & $52 \%$ & 38 & $37 \%$ & 0 & $0 \%$ & 104 & $100 \%$ \\
En casa & 6 & $25 \%$ & 13 & $54 \%$ & 5 & $21 \%$ & 0 & $0 \%$ & 24 & $100 \%$ \\
Otros & 8 & $16 \%$ & 26 & $53 \%$ & 15 & $31 \%$ & 0 & $0 \%$ & 49 & $100 \%$ \\
\hline Total & 48 & $12 \%$ & 202 & $51 \%$ & 146 & $37 \%$ & 1 & $0 \%$ & 397 & $100 \%$ \\
\hline Fuente: Encuesta sobre nivel de conocimiento de cultura ambiental & & & & & & & &
\end{tabular}

La tabla $\mathrm{N} .^{\circ} 5$, muestra el nivel de conocimientos sobre 'cultura ambiental' que presentan los habitantes del Distrito de Wánchaq es mayormente 'medio - alto', destacando los que trabajan en sectores privados en un $92 \%$, seguido por los que trabajan de manera independiente en un $89 \%$.

Tabla N. ${ }^{\circ} 6$ DESCRIPCIÓN SOBRE EL NIVEL DE CULTURA AMBIENTAL REALIZADO EN SUS TRES COMPONENTES POR LOS HABITANTES DEL DISTRITO DE WANCHAQ - CUSCO

\begin{tabular}{lcccccc}
\hline \multirow{2}{*}{$\begin{array}{c}\text { Nivel de } \\
\text { conocimiento }\end{array}$} & \multicolumn{2}{c}{ Agua } & \multicolumn{2}{c}{ Aire } & \multicolumn{2}{c}{ Suelo } \\
\cline { 2 - 7 } & $\mathrm{N}^{\circ}$ & $\%$ & $\mathrm{~N}^{\circ}$ & $\%$ & $\mathrm{~N}^{\circ}$ & $\%$ \\
\hline Muy bajo & 12 & 3 & 1 & 0.3 & 13 & 3.3 \\
Bajo & 61 & 15.4 & 61 & 15.4 & 46 & 11.6 \\
Medio & 157 & 39.5 & 248 & 62.5 & 135 & 34 \\
Alto & 150 & 37.8 & 81 & 20.4 & 197 & 49.6 \\
Muy alto & 17 & 4.3 & 6 & 1.5 & 6 & 1.5 \\
\hline Total & 397 & 100 & 397 & 100 & 397 & 100 \\
\hline
\end{tabular}

Fuente: Encuesta sobre nivel de conocimiento de cultura ambiental 
Esta tabla comparativa $\mathrm{N} .^{\circ}$ 6, muestra que el nivel de conocimientos sobre el agua, aire y suelo que tienen los habitantes del Distrito de Wánchaq es 'mayormente medio' en una proporción del 39,5 \% y 62,5 \% respectivamente; sin embargo, los conocimientos respecto al suelo son mayormente altos reflejado por el 49,6\% de la muestra investigada.

\begin{tabular}{|c|c|c|c|}
\hline & \multicolumn{3}{|c|}{ WÁNCHAQ - CUSCO } \\
\hline & Conocimiento & $\mathrm{N} .^{\circ}$ & $\%$ \\
\hline & Bajo & 48 & 12,1 \\
\hline & Medio & 202 & 50,9 \\
\hline & Alto & 146 & 36.8 \\
\hline & Muy alto & 1 & 0,3 \\
\hline & Total & 397 & 100.0 \\
\hline
\end{tabular}

Fuente: Encuesta sobre nivel de conocimiento de cultura ambiental

La tabla $\mathrm{N}^{\circ}{ }^{\circ}$, muestra que el nivel de conocimientos sobre cultura ambiental de la mayoría de los habitantes del distrito de Wánchaq es medio, reflejado por 202 habitantes en una proporción del 50,9\% de un total de 397 habitantes participantes de la investigación; sin embargo, también hay una porcentaje importante de habitantes que presentan conocimientos altos respecto al tema de investigación.

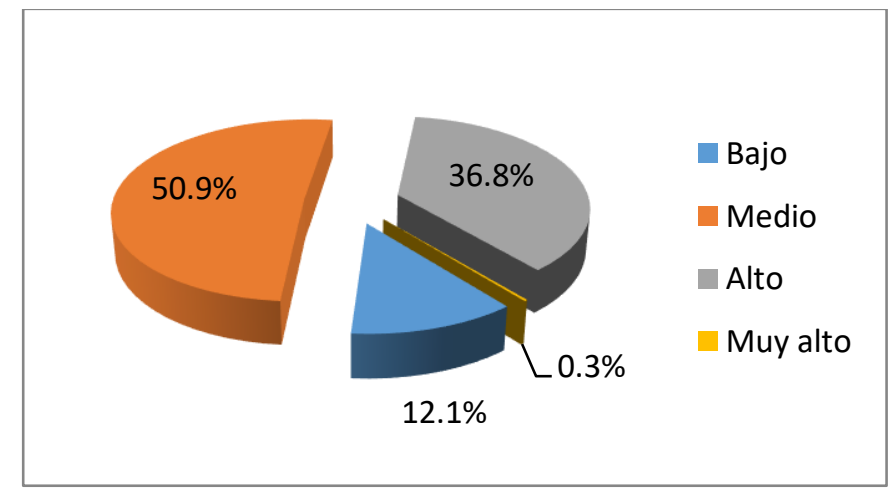

Figura N. $^{\circ} 1$ Descripción sobre el nivel de cultura ambiental por los habitantes del distrito de Wánchaq - Cusco 


\section{DISCUSIÓN}

La investigación estuvo orientada a conocer el nivel de cultura ambiental de los habitantes del Distrito de Wánchaq, uno de los 8 de la Región Cusco, considerando la conservación en tres indicadores: agua, aire y suelo.

Para la investigación se elaboró una encuesta, la que fue aplicada a una muestra de 397 personas, con preguntas generales referidas a edad, sexo, nivel educativo y lugar de trabajo; luego se formulan 60 interrogantes, de las cuales 20 se refieren a cada uno de los indicadores. El área geográfica de estudio está emplazado al sureste de la ciudad de Cusco, su población al año 2007 fue de 59 134, (Censo 2007). La localidad está constituida por jóvenes entre 15 a 32 años: 43,3 \% (172 individuos), y los que se ubican entre los 33 a 50 años 37,5 \% (149 individuos), alcanzando entre ambos un $80,8 \%$.

Los datos obtenidos por nuestro trabajo indican que se trata de un distrito con población joven, con nivel de conocimiento medio alto sobre cultura ambiental y respecto a la educación, se aprecia que 324 personas tienen nivel educativo superior, haciendo un 81,6\% y 8 técnicos que constituyen el $2 \%$, sumando un total de $84 \%$. Un porcentaje menor $16 \%$ tienen nivel secundario y solo una persona tiene nivel primario, por lo que se aprecia que es una localidad que alcanza un nivel medio. De acuerdo al censo de población y vivienda de 2007, esta jurisdicción muestra que el 50 \% de sus habitantes tienen educación superior, que va de incompleta a completa; en comparación a los que se encuentran en las inmediaciones de Cusco, como son: San Sebastián con 27 \%, San Jerónimo 27,50 \%, Saylla 15 \%, entre otros. Asimismo, en el cuadro N. ${ }^{\circ}$, sobre desarrollo humano en la provincia del Cusco. El Distrito de Wánchaq, frente a los demás, muestra los mayores niveles en las categorías de 'desarrollo humano', 'esperanza de vida al nacer', 'alfabetismo', 'escolaridad', 'logro educativo' e 'ingreso familiar'. Lo mencionado significa una fortaleza para este sector de la población de la Región Cusco, que se puede convertir en un modelo de gestión desde la misma sociedad civil respecto al cuidado del ambiente, tal como lo menciona (Barrios, 2010) cuando trata sobre los ecosistemas urbanos.

En el aspecto del conocimiento, los datos que aparecen en el cuadro $\mathrm{N} .^{\circ} 2$, arriba mencionado, muestra que los niveles de percepción, respecto al ambiente y los alcanzados con la investigación, están relacionados con la actitud y el comportamiento de los habitantes de este distrito — referidos a la cultura ambiental — que fue la primera interrogante que nos 
habíamos planteado en el trabajo efectuado y que se muestra con claridad en el mayor cuidado que ponen en sus áreas verdes y otros espacios libres, apreciándose vegetación arbustiva propia de la zona, plantas ornamentales y el cuidado de las fachadas de los edificios, aspectos externos que nos indican el interés que cada propietario o vecino tiene sobre su inmueble y su entorno.

Considerando todo ello, el distrito ostenta desde hace varias décadas, la denominación de «Distrito Jardín». A esto debemos añadir el tratamiento de los residuos sólidos que ha permitido la internalización de cada persona, especialmente de las amas de casa, en el manejo de los desechos, haciendo que cada familia separe estos en bolsas diferentes, las mismas que son recogidas por la institución «Proyecto Panti», por lo que afirmamos que tienen conocimiento sobre cultura ambiental que se manifiesta en su comportamiento referido a la conservación del suelo, habiendo alcanzado — de acuerdo a nuestro estudio-, el nivel alto respecto a nuestro indicador y sub indicador. Esto corrobora lo indicado por el PNUD respecto al desarrollo humano, el mismo que está sustentado en tres parámetros: salud, educación, riqueza o economía; hecho que claramente se refleja en el cuadro mencionado. Resumiendo, indicamos que los aprendizajes adquiridos por las personas de manera tácita o inconsciente, e intencional a través de una institución, demuestran el juicio de valor que alcanzan sobre el cuidado respecto al ambiente y su aplicación manifiestas en las actitudes y comportamiento demostrados; aspectos que determinan un nivel de cultura ambiental: que es justamente lo que se requiere si pretendemos contar con una adecuada calidad de vida. Creemos conveniente indicar que la localidad en estudio es una población que resultó a consecuencia del sismo de 1950, donde se reubicaron a las personas que fueron afectadas y se trataba de un grupo constituido por familias con nivel educativo superior, por ende, su cultura es, sobre todo, citadina.

Si comparamos este distrito con otros que están alejados del centro de la ciudad, seguramente encontraremos diferencias en su cultura y sus actitudes frente al medio ambiente, por cuanto, muchos son producto de la migración del campo a la ciudad y, por tal razón, desarrollan algunas de sus actividades diarias de acuerdo a sus vivencias y localismos plasmados en sus costumbres, tradiciones, creencias y valores; argumentos que también fueron considerados por (Miranda Murillo, 2013). Si los animales inferiores cuidan su espacio geográfico, ¿por 
qué, nosotros los humanos que somos creativos, adaptativos y flexibles, no podemos mejorar nuestro entorno y tratar de reducir nuestras acciones negativas contra el ambiente?

Hoy en día estamos atravesando una crisis ambiental que afecta a todos los seres vivos, apreciamos la escasez del líquido elemento dulce, su contaminación, así como del aire y el suelo, el deterioro de la capa de ozono; el cambio climático, la deforestación, pérdida de biodiversidad y suelo fértil, entre otros, preocupación que es manifestada por (GORDILLO MARTÍNEZ, Alberto José, CABRERA CRUZ, René Bernardo Elías, HERNÁNDEZ MARIANO, Marisol, GALINDO, Erick, OTAZO, Elena, \& PRIETO, Francisco, 2010).

A lo indicado debemos añadir el calentamiento global producido por la emisión de gases de invernadero, cuyas complicaciones las estamos viviendo. Ante estos problemas (Roselló, Tamara y Del Toro, Marielys, 2006, pág. 40), manifiestan que no nos podemos quedar de brazos cruzados y se debe alzar la voz para un llamado de alerta, para enseñar mejores hábitos y conductas ambientalmente adecuadas y desde nuestro trabajo vemos que efectivamente la población en estudio tiene hábitos encaminados a lograr dicho propósito respecto al agua, aire y suelo; habiendo alcanzado el nivel de medio en los dos primeros indicadores, y de alto en el último. También apreciamos en otras esferas — como en el caso de México- que se han realizado inventarios en municipios sobre la contaminación al suelo, agua y aire, estudio presentado por (Cabrera Cruz, René Bernardo E., Gordillo Martínez, Alberto José y Cerón Beltrán, Álvaro, 2003).

Los porcentajes obtenidos por nuestro trabajo se pueden mejorar con acciones de sensibilización que la misma sociedad civil y las instituciones públicas y privadas pueden ejecutar, con el objeto de cambiar actitudes y comportamientos ambientales arraigados en la cultura de los pueblos, como lo manifiesta Miranda (2013, Óp. Cit., p. 30).

Entendemos a la conservación como el cuidado y la defensa que se realice con anticipación del ambiente o entorno donde vivimos, para evitar su deterioro. Es también efectuar una serie de acciones para proteger y salvaguardar los valores materiales, culturales y sociales que existen en un lugar y que son parte de la vida del hombre puesto que es necesario hacerlo sostenible para las generaciones futuras, incluye el espacio geográfico, el agua, aire, suelo; así como las especies animales y vegetales existentes y la relación entre ellos. (Muñoz Guzmán, 2010), cuando trata sobre la conservación del medio ambiente, manifiesta que el hombre no es un ser aislado en este planeta sino, por el contrario, interactúa con otros seres vivos que tienen la misma necesidad de subsistir, razón por la cual el aire, el agua, el clima 
adecuado, etc., son trascendentes para el equilibrio entre los seres vivos y los factores fisicoquímicos. Esta necesidad de conservación, se manifiesta en el conocimiento que sobre ella tienen los habitantes del Distrito de Wánchaq y que viene a constituir su cultura ambiental.

La sociedad contemporánea se está convirtiendo en artífice de su propia destrucción por cuanto, en la actualidad se libera cantidad significativa de sustancias tóxicas a la atmósfera, hecho que genera cambios en el clima y la contaminación del agua y el aire, haciendo que el primero se convierta en un recurso finito.

En ese entender, es urgente realizar diferentes acciones, muchas de ellas inclusive simples, para proteger y conservar nuestro planeta, inculcar en cada persona a través de las diferentes formas de comunicación, campañas de sensibilización sobre una adecuada cultura ambiental, así lo manifiestan Roselló y Del Toro (2005, Óp. Cit., p, 60), cuando mencionan la necesidad de impulsar ejes comunicativos que permitan un diálogo sobre la problemática ambiental e incentivar a la creatividad de acciones para producir mecanismos de equilibrio con la naturaleza, tal como el que nosotros proponemos con la ejecución de un «proyecto de capacitación» orientado a sensibilizar a la población objeto de estudio.

(Castillo León, 2009), indica que la relación directa naturaleza-sociedad, para armonizarla se debe tener en cuenta que la cultura ambiental en la conservación del medio se convierte, en parte indispensable de lo cognoscitivo de un ser social integrado a una comunidad; Guadarrama y Pelegrín (1990, Óp. Cit., p. 23), manifiestan que la cultura ambiental se expresa en esa segunda naturaleza construida por el hombre, las relaciones no solo materiales, sino también espirituales que se establecen formando parte de ese concepto grande que es el "medio ambiente". Al ser la cultura todo aquello creado por el hombre, su herencia social, esta se va formando a través del tiempo y en ello participan sus conocimientos, experiencia, educación, género, edad; en relación directa con su entorno.

También es necesario considerar que es un proceso participativo que busca despertar conciencia e identificación en la población con la problemática ambiental, de manera general y específica. Por ello, a través de distintas estrategias — como la práctica cotidiana de formas de comportamiento - y la educación no formal y formal, que es el motor de transformación social, permite que se transmita la cultura para hacerla sostenible y se encamine para lograr 
la conservación del ambiente. Nuestro estudio ha demostrado, en lo referente a la educación formal, que se alcanzó el nivel medio referido a la conservación del agua y el aire.

Otros aspectos que son interesantes a tomar en cuenta para la formación de una cultura ambiental o ecológica, son las actitudes que asumen las personas respecto a su ambiente. (Yarlequé Chocas, 2004, pág. 75), en su estudio sobre «Actitudes hacia la conservación ambiental en estudiantes de educación secundaria», en la costa, sierra y selva del Perú, ha llegado a la conclusión que quienes habitan en la costa, muestran actitudes favorables a la conservación ambiental, además, manifiesta que las mujeres tienen actitudes más favorables que los varones respecto al agua. Los resultados de nuestra investigación nos han permitido concluir que también es el género femenino el que ha alcanzado el nivel medio, con $52 \%$, en comparación al masculino que lograron el 50\%. Esto indica que existe una ligera diferencia, por cuanto sus actividades cotidianas las pone más a menudo en contacto con los elementos que plantea nuestro estudio, en especial el agua.

Los datos referidos al aire indican que los varones alcanzan el nivel medio con 131 individuos, con el $64 \%$, y las mujeres con 117 individuos con el $61 \%$. De lo afirmado podemos colegir que, en la actualidad en el distrito existe un parque industrial que no cuenta con los controles adecuados referidos a la contaminación del suelo y el aire. En este lugar se halla la mayor cantidad de talleres de mecánica automotriz los que contaminan el suelo y el aire y que son percibidos mayoritariamente por los varones que se encargan del cuidado y mantenimiento de sus vehículos y, con referencia a las mujeres, no es que ellas estén ajenas a esta realidad, sino que no enfatizaron en los componentes mencionados al responder la encuesta.

De manera general, sobre la cultura ambiental de los habitantes del Distrito de Wánchaq de la Región Cusco, ha alcanzado el nivel medio con un $51 \%$; lo que demuestra que sí efectivamente conocen sobre los problemas ambientales que nos afectan y realizan acciones encaminadas a su protección.

(Badillo y Romero 2012) mencionan, que es necesario entender la sensibilización y creación de una cultura ambiental en trabajadores, contratistas y empleados del campo como un proceso para llegar a minimizar todos los impactos ambientales significativos utilizando la comunicación y la capacitación en todos los niveles de la sociedad, para crear acciones proteccionistas y a la utilización adecuada de los recursos, evitando contaminar el aire y el 
agua, así también lo menciona (Meraz López, 2003) en su trabajo sobre «Cultura Ecológica para el cuidado del ambiente en una empresa dedicada a la fabricación de enseres domésticos», concluye que es necesario capacitar al personal de las empresas que producen bienes para crear una cultura ecológica, por cuanto existe un compromiso con la sociedad y el respeto a la naturaleza con cuyos estudios estamos de acuerdo. El trabajo estuvo encaminado a la concientización de la población en el control ambiental. La encuesta que hemos aplicado a los pobladores del Distrito de Wánchaq, respecto al lugar de trabajo y su relación con la cultura ambiental, demostró que las personas que trabajan en el sector privado alcanzaron el 92\%, seguidos de los independientes que lograron el $89 \%$, siendo su nivel medio alto, dato obtenido después de aplicar el instrumento de evaluación y que nos está demostrando que se trata de un grupo humano con nivel educativo superior y técnico que están realizando acciones de protección del ambiente, tanto en su vivienda como en su centro de trabajo.

Considerando lo indicado por los autores mencionados que coinciden en realizar acciones de capacitación y comunicación para minimizar los impactos ambientales, nosotros en esa línea - ya se ha mencionado párrafos anteriores - propiciamos la ejecución del proyecto de capacitación y sensibilización hacia la comunidad y la distribución de cartillas en las viviendas, porque creemos que a mayor información se acrecentará la conciencia ambiental. El estudio realizado en el Distrito de Wánchaq, de la Provincia y Región Cusco, sobre el conocimiento de la cultura ambiental, ha dado como resultado que tienen un nivel medio, lo que indica que la población está imbuida de la problemática ambiental y realiza acciones en su vida cotidiana para contar con un ambiente sano. Este nivel mostrado por nuestro trabajo debía optimizarse y, para dicho propósito, se debe realizar diferentes actividades referidas con la concientización desde el hogar, el colegio y otros espacios.

Finalmente, la investigación se ha enfocado en el estudio de la cultura ambiental desde los elementos básicos del ambiente como el agua, aire y suelo, analizados desde las categorías de edad, género, educación y lugar de trabajo; habiendo permitido alcanzar con mayor aproximación el conocimiento sobre dichos aspectos que tiene el distrito en estudio. 


\section{SUGERENCIAS}

- Invitar a otros estudiosos a seguir investigando el tema para conseguir un ambiente saludable, hacer de conocimiento de las autoridades que manejan una ciudad, para que en sus planes de desarrollo regional y distrital definan políticas ambientales, considerando que el mundo está atravesando cambios drásticos que está afectando a nuestro derecho a una vida sana.

- Que el área de medio ambiente del municipio distrital incluya y desarrolle programas de capacitación y sensibilización para diferentes niveles etarios de la sociedad civil, orientados a lograr un ambiente sano.

- Propiciar que la prensa hablada, escrita y televisiva, puedan pasar pequeños avisos respecto a temas de protección al medio ambiente, sin necesidad que por dicha acción requieran de una paga, muy por el contrario: como entidades sensibles por la protección a nuestro medio ambiente.

- Alentar para que los niños y la juventud realicen tareas de limpieza en sus barrios y, enseñar las tres R: Reducir, Reutilizar, Reciclar; a través de cursos sobre manejo de residuos sólidos. 


\section{BIBLIOGRAFÍA}

Amezcua, Cardelia y Prodanza, C. (2006). Comunidades para la esperanza: Manual básico de cultura ambiental infantil a través del arte y la Carta a la Tierra. Eronguaricaro, 59.

Barrios, J. C. (2010). Ecosistemas urbanos. Ambienta, s/p. Obtenido de http://www.revistaambienta.es/WebAmbienta/marm/Dinamicas/secciones/articulos/Urb anos.htm

Bonfil Batalla, G. (2004). Manual Básico del Promotor Cultural (5.ta. Ed. (1.ra 1998) ed.). México DF, México: Edit. Tlallocan.

Brundtland, G. H. (1987). Report of the World Commission on Environment and Development: Our Common Future. Nueva York.: Naciones Unidas.

Cabrera Cruz, René Bernardo E., Gordillo Martínez, Alberto José y Cerón Beltrán, Álvaro. (19 de 4 de 2003). Inventario de contaminación emitida a suelo, agua y aire en 14 municipios del Estado de Hidalgo, México. Revista Internacional de Contaminación Ambiental, 171-181. Obtenido de http://ccaunam.atmosfcu.unam.mx/editorial/rica/acervo/vol_19_4/2.pdf

Carrasco Arroyo, S. (2006). Medir la cultura: Una tarea inacabada. Periférica(7), 26. doi:ISSN -15771172

Castillo León, Y. (2 de setiembre de 2009). Cultura ambiental en el diagnóstico comunitario. Recuperado el 2017, de https://www.gestiopolis.com/cultura-ambiental-diagnosticocomunitario/: https://www.gestiopolis.com/cultura-ambiental-diagnostico-comunitario/

Cuervo Álvarez, B. (2012). El medio ambiente y su destrucción. Oviedo, Asturias, España. Obtenido de http://www.monografias.com/trabajos90/medio-ambiente-y-su-destruccion/medioambiente-y-su-destruccion.shtml

Cusco, R. (2012). Informe Regional. Región Cusco. Cusco: s/n. Obtenido de www.Región Cusco. Gob.pe/transparencia 2012

Española, A. d. (2014). Diccionario de la lengua española. Madrid: Alfaguara.

Giannuzzo, A. N. (2010). Los estudios sobre el ambiente y la ciencia ambiental. Scientiae Studia: Revista Latinoamericana de Filosofía e Historia da Ciencia, 48. doi: http://dx.doi.org/10.1590/S1678-31662010000100006

GORDILLO MARTíNEZ, Alberto José, CABRERA CRUZ, René Bernardo Elías, HERNÁNDEZ MARIANO, Marisol, GALINDO, Erick, OTAZO, Elena, \& PRIETO, Francisco. (26 de 3 de 2010). Evaluación regional del impacto antrópico sobre aire, agua y suelo. Caso: huasteca hidalguense, México. Revista internacional de contaminación ambiental. Recuperado el 02 de septiembre de 2017, 229-251. Obtenido de http://www.scielo.org.mx/scielo.php?script=sci_arttext\&pid=S018849992010000300006\&lng=es\&tlng=es.

Guadarrama, P y N. Pelegrín. (1990). Lo universal y lo específico en la cultura. La Habana: Editorial Ciencias Sociales. 
Herskovits, M. J. (2000). El hombre y sus obras. México: Fondo de Cultura Económica.

Isaac-Márquez, Ricardo; Salavarría García, Oswaldo Orlando; Eastmond Spencer, Amarella; Ayala Arcipreste, María Esther; Arteaga Aguilar, Marco Antonio; Et. Al. (2011). Cultura ambiental en estudiantes de bachillerato. Estudio de caso de la educación ambiental en el nivel medio superior de Campeche. REDIE. Revista Electrónica de Investigación Educativa, , 8399.

Mac Gregor, J. (Setiembre de 2005). Reflexiones en torno a la identidad. PACA EP, 51.

Mata, A. (2004). La transformación de la cultura ambiental mediante la docencia universitaria. Revista Biocenosis, "/vol.18(1-2)... P. 78. Obtenido de n.d.www.cca.ucr.ac.cr/ccawebanterior/sites/default/files/cv-mata-segreda.pdf

Medina, H. (2015). Factores determinantes del nivel de contaminación del aire con monóxido de carbono en la ciudad del Cusco-2014. YAchay. Revista científico cultural de la Universidad Andina del Cusco, IV, 21-26.

Meraz López, P. A. (2003). Cultura ecológica para el cuidado del medio ambiente en una empresa dedicada a la fabricación de enseres domésticos. Tesis de Maestría, Universidad Autónoma de Nuevo León, México. Obtenido de http://eprints.uanl.mx/5440/

Miranda Murillo, L. M. (2013). Cultura ambiental: un estudio desde las dimensiones de valor, creencias, actitudes y comportamientos ambientales. Producción + limpia, 8(2). Obtenido de http://repository.lasallista.edu.co:8080/ojs/index.php/pl/article/view/527

Miranda, L. (2011). Cultura ambiental: un estudio desde las dimensiones de valor, creencias, actitudes y comportamientos ambientales. Tolima: Universidad del Tolima.

Muñoz Guzmán, M. A. (2010). Modelo Económico Mundial y la Conservación del Medio Ambiente. (U. C. Colon, Ed.) Veracruz , MEXICO. Obtenido de Edición electrónica. Texto completo en http://eumed.net/cursecon/libreria/index.htm

Romero, S. (2012). Fortalecimiento de la cultura ambiental al área asociada a campo escuela colorado . (U. I. Petróleos, Ed.) Bucaramanga, Colombia. Obtenido de http://tesis.ucsm.edu.pe/repositorio/bitstream/handle/UCSM/5988/9I.0361.DR.pdf?sequ ence $=1 \&$ isAllowed $=\mathrm{y}$

Roselló, Tamara y Del Toro, Marielys. (2006). Perspectiva comunitaria en busca de una cultura ambiental, Educación Popular y Educación Popular Ambiental. ¿Un posicionamiento Único? La Habana, Cuba: Editorial Caminos.

Seoanez Calvo, M. (1999). Contaminación del suelo: Estudios, tratamiento y gestión. Madrid: Mundi Prensa S.A. Obtenido de https://www.casadellibro.com/libro-contaminacion-delsuelo-estudios-tratamiento-y-gestion/9788471148063/641567

Silva Arroyave, Sandra y Correa Restrepo,Francisco (2009) Análisis de la contaminación del suelo: revisión de la normativa y posibilidades de regulación económica http://www.scielo.org.co/pdf/seec/v12n23/v12n23a2.pdf Medellin Colombia Pag. 15. 
UNESCO. (2012). "La cultura", memoriavirtualbucaramanga.com/definición-cultura Unesco/. Informe-Memoria, Bucaramanga. Obtenido de https://www.google.com.pe/?gws_rd=ssl\#q=lilia+a+albert+contaminacion+ambiental. P. 53

Wánchaq, M. d. (2013). Plan de desarrollo concertado de la Municipalidad distrital de Wanchaq. Cusco. Cusco: Municipalidad de Wánchaq.

Winick, C. (1989). Diccionario de Antropología. Buenos Aires, Argentina: Edit. Troquel.

Yarlequé Chocas, L. A. (2004). Actitudes hacia la conservación ambiental en estudiantes de educación secundaria. Tesis de grado. Lima, Perú: Universidad Nacional Mayor de San Marcos. Obtenido de http://cybertesis.unmsm.edu.pe/bitstream/cybertesis/561/1/Yarleque_cl.pdf 